\title{
EXISTENCE AND ANALYTICITY OF LEI-LIN SOLUTION TO THE NAVIER-STOKES EQUATIONS
}

\author{
HANTAEK BAE \\ (Communicated by Walter Craig)
}

ABSTRACT. In this paper, we prove the recent work of Lei-Lin in a slightly different setting, which enables us to prove analyticity of the solution.

\section{INTRODUCTION}

In this paper, we study the incompressible Navier-Stokes equations in $\mathbb{R}^{3}$ :

$$
v_{t}+v \cdot \nabla v-\mu \Delta v+\nabla p=0, \quad \nabla \cdot v=0,
$$

where $v$ is the velocity field, $p$ is the pressure, and $\mu>0$ is the viscosity coefficient.

The aim of this paper is to prove the recent work of Lei-Lin [15] in a slightly different fashion. To this end, we reformulate the problem as follows. Since $\nabla \cdot v=0$, we can rewrite (1.1) by projecting it onto the divergence-free space. Namely, we apply the orthogonal projection $\mathbb{P}=I d-\nabla(-\Delta)^{-1}$ div in $L^{2}$ over divergence-free vector fields to (1.1). Then, we obtain

$$
v_{t}+\mathbb{P} \nabla \cdot(v \otimes v)-\mu \Delta v=0 .
$$

Formally, we can express a solution $v$ of (1.2) in the integral form:

$$
v(t)=e^{\mu t \Delta} v_{0}-\int_{0}^{t}\left[e^{\mu(t-s) \Delta} \mathbb{P} \nabla \cdot(v \otimes v)(s)\right] d s .
$$

Any solution satisfying this integral form is called a mild solution, and we can find it by using a fixed point argument for the function $v \mapsto F(v)$, where

$$
F(v)(t)=e^{\mu t \Delta} v_{0}-\int_{0}^{t}\left[e^{\mu(t-s) \Delta} \mathbb{P} \nabla \cdot(v \otimes v)(s)\right] d s .
$$

The function spaces for solving this integral equation correspond to a scale invariance property of the equation. Assume that $(v, p)$ solves (1.1). Then, the same is true for rescaled functions:

$$
v_{\lambda}(t, x)=\lambda v\left(\lambda^{2} t, \lambda x\right), \quad p_{\lambda}(t, x)=\lambda^{2} p\left(\lambda^{2} t, \lambda x\right) .
$$

Under these scalings, $L^{3}, \dot{H}^{\frac{1}{2}}, \dot{W}^{\frac{3}{p}-1, p}, \dot{B}_{p, q}^{\frac{3}{p}-1}$, and $B M O^{-1}$ are critical spaces for initial data $(t=0)$ to name a few, and one can find various well-posedness results for small data in these critical spaces in $[3,6,8,13,14,20$.

Received by the editors May 5, 2013.

2000 Mathematics Subject Classification. Primary 35Q30, 76D03.

Key words and phrases. Navier-Stokes equations, analyticity of mild solutions. 
We now state the result in [15], where Lei and Liu introduced the following function spaces:

$$
\begin{aligned}
& X^{-1}=\left\{f \in \mathscr{D}^{\prime}\left(\mathbb{R}^{3}\right): \int_{\mathbb{R}^{3}}|\xi|^{-1} \hat{f}(\xi) \mid d \xi<\infty\right\}, \\
& X^{1}=\left\{f \in \mathscr{D}^{\prime}\left(\mathbb{R}^{3}\right): \int_{\mathbb{R}^{3}}|| \xi|\hat{f}(\xi)| d \xi<\infty\right\},
\end{aligned}
$$

where $X^{-1}$ is also a critical space for initial data.

Theorem 1.1 ([15]). For any initial data in $X^{-1}$ with $\left\|v_{0}\right\|_{X^{-1}}<\mu$, there exists a unique global in time solution $v \in C\left(\mathbb{R}_{+} ; X^{-1}\right) \cap L^{1}\left(\mathbb{R}_{+} ; X^{1}\right)$ such that

$$
\sup _{0 \leq t<\infty}\left[\|v(t)\|_{X^{-1}}+\left(\mu-\left\|v_{0}\right\|_{X^{-1}}\right) \int_{0}^{t}\|\nabla v(s)\|_{L^{\infty}} d s\right] \leq\left\|v_{0}\right\|_{X^{-1}} .
$$

In this paper, we prove a similar result to [15] in the following time dependent spaces:

$$
\begin{aligned}
& \mathscr{X}^{-1}=\left\{f \in \mathscr{D}^{\prime}\left(\mathbb{R}_{+} \times \mathbb{R}^{3}\right): \int_{\mathbb{R}^{3}}\left[\left.\sup _{0 \leq t<\infty}|| \xi\right|^{-1} \hat{f}(t, \xi) \mid\right] d \xi<\infty\right\}, \\
& \mathscr{X}^{1}=\left\{f \in \mathscr{D}^{\prime}\left(\mathbb{R}_{+} \times \mathbb{R}^{3}\right): \int_{\mathbb{R}^{3}}\left[\int_{0}^{\infty}|| \xi|\hat{f}(t, \xi)| d t\right] d \xi<\infty\right\} .
\end{aligned}
$$

We note that we define these spaces by switching the order of time and Fourier variables to prove the existence result (Theorem 1.2) in the integral form (1.3). Although we cannot take the size of initial data precisely in terms of the size of the viscosity coefficient, our result allows us to establish analyticity of the solution (Theorem 1.3). (For various analyticity results, see [1, 2, 7, 10, 12, 18, 19] and the references therein.) For simplicity, we take $\mu=1$ for the rest of the paper.

Theorem 1.2. There exists a positive constant $\epsilon_{0}>0$ such that for any initial data in $X^{-1}$ with $\left\|v_{0}\right\|_{X^{-1}}<\epsilon_{0}$ there exists a unique global in time solution $v \in$ $\mathscr{X}^{-1} \cap \mathscr{X}^{1}$ such that

$$
\|v\|_{\mathscr{X}^{-1}}+\|v\|_{\mathscr{X}^{1}} \lesssim\left\|v_{0}\right\|_{X^{-1}}
$$

Theorem 1.3. There exists a positive constant $\epsilon_{0}>0$ such that for any initial data in $X^{-1}$ with $\left\|v_{0}\right\|_{X^{-1}}<\epsilon_{0}$, the solution in Theorem 1.2 is analytic in the sense that

$$
\left\|e^{\sqrt{t}|D|} v\right\|_{\mathscr{X}^{-1}}+\left\|e^{\sqrt{t}|D|} v\right\|_{\mathscr{X}^{1}} \lesssim\left\|v_{0}\right\|_{X^{-1}},
$$

where $e^{\sqrt{t}|D|}$ is a Fourier multiplier whose symbol is given by $e^{\sqrt{t}|\xi|}$.

\section{Proof of Theorem 1.2}

Here we provide only a priori estimate (1.7). The iteration can be performed by a regularization argument and we skip the iteration step.

Step 1. We first take the Fourier transform to (1.3):

$$
\begin{aligned}
\widehat{v}(t, \xi) & =e^{-t|\xi|^{2}} \widehat{v_{0}}(\xi) \\
& -\int_{0}^{t}\left[e^{-(t-s)|\xi|^{2}} \mathbb{P} \xi \cdot\left(\int_{\mathbb{R}^{3}} \widehat{v}(s, \xi-\eta) \widehat{v}(s, \eta) d \eta\right)\right] d s .
\end{aligned}
$$


Since the Fourier multiplier $\mathbb{P}$ is a bounded Fourier multiplier, we ignore this term in the estimations. We first estimate $v$ in $\mathscr{X}^{-1}$. By multiplying (2.1) by $|\xi|^{-1}$, we have

$$
\begin{aligned}
\left.|| \xi\right|^{-1} \widehat{v}(t, \xi) \mid & \leq\left. e^{-t|\xi|^{2}}|| \xi\right|^{-1} \widehat{v_{0}}(\xi) \mid \\
& +\int_{0}^{t}\left[e^{-(t-s)|\xi|^{2}}\left(\int_{\mathbb{R}^{3}}|\widehat{v}(s, \xi-\eta)||\widehat{v}(s, \eta)| d \eta\right)\right] d s .
\end{aligned}
$$

Since $1 \leq \frac{|\xi-\eta|}{|\eta|}+\frac{|\eta|}{|\xi-\eta|}$, we can rewrite the nonlinear term as follows:

$$
\begin{aligned}
& \int_{0}^{t}\left[\left(\int_{\mathbb{R}^{3}}|\widehat{v}(s, \xi-\eta)||\widehat{v}(s, \eta)| d \eta\right)\right] d s \\
& \leq \int_{0}^{t}\left[\left(\int_{\mathbb{R}^{3}}\left(\frac{|\xi-\eta|}{|\eta|}+\frac{|\eta|}{|\xi-\eta|}\right)|\widehat{v}(s, \xi-\eta)||\widehat{v}(s, \eta)| d \eta\right)\right] d s .
\end{aligned}
$$

We take the $L^{\infty}$ norm in time to (2.2). Then,

$$
\begin{aligned}
& \left.\sup _{0 \leq t<\infty}|| \xi\right|^{-1} \widehat{v}(t, \xi) \mid \\
& \leq\left.|| \xi\right|^{-1} \widehat{v_{0}}(\xi) \mid \\
& +\int_{0}^{\infty}\left[\left(\int_{\mathbb{R}^{3}}\left(\frac{|\xi-\eta|}{|\eta|}+\frac{|\eta|}{|\xi-\eta|}\right)|\widehat{v}(t, \xi-\eta)||\widehat{v}(t, \eta)| d \eta\right)\right] d t \\
& \left.\lesssim|| \xi\right|^{-1} \widehat{v_{0}}(\xi) \mid+\left[\int_{0}^{\infty}|\xi| \widehat{v}(t, \xi) d t\right] *_{\xi}\left[\left.\sup _{0 \leq t<\infty}|| \xi\right|^{-1} \widehat{v}(t, \xi) \mid\right] .
\end{aligned}
$$

We take the $L^{1}$ norm in $\xi$ to (2.4). By Young's inequality,

$$
\begin{array}{r}
\|v\|_{\mathscr{X}^{-1}} \lesssim\left\|v_{0}\right\|_{X^{-1}}+\|v\|_{\mathscr{X}^{1}} \\
\mid v \|_{\mathscr{X}^{-1}} .
\end{array}
$$

Step 2. We next estimate $v$ in $\mathscr{X}^{1}$. We multiply (2.1) by $|\xi|$. Then,

$$
\begin{aligned}
\| \xi|\widehat{v}(t, \xi)| & \leq\left.|\xi|^{2} e^{-t|\xi|^{2}}|| \xi\right|^{-1} \widehat{v_{0}}(\xi) \mid \\
& +\int_{0}^{t}\left[|\xi|^{2} e^{-(t-s)|\xi|^{2}}\left(\int_{\mathbb{R}^{3}}|\widehat{v}(s, \xi-\eta)||\widehat{v}(s, \eta)| d \eta\right)\right] d s .
\end{aligned}
$$

As before, we rewrite the nonlinear term as (2.3) and we take the $L^{1}$ norm in time to (2.7). Using

$$
\int_{0}^{\infty}|\xi|^{2} e^{-t|\xi|^{2}} d t<C
$$

and Young's inequality, we have

$$
\begin{aligned}
& \int_{0}^{\infty}|| \xi|\widehat{v}(t, \xi)| d t \\
& \leq\left.|| \xi\right|^{-1} \widehat{v_{0}}(\xi) \mid \\
& +\int_{0}^{\infty}\left[\left(\int_{\mathbb{R}^{3}}\left(\frac{|\xi-\eta|}{|\eta|}+\frac{|\eta|}{|\xi-\eta|}\right)|\widehat{v}(t, \xi-\eta)||\widehat{v}(t, \eta)| d \eta\right)\right] d t \\
& \left.\lesssim|| \xi\right|^{-1} \widehat{v_{0}}(\xi) \mid+\left[\int_{0}^{\infty}|\xi| \widehat{v}(t, \xi) d t\right] * \xi\left[\left.\sup _{0 \leq t<\infty}|| \xi\right|^{-1} \widehat{v}(t, \xi) \mid\right] .
\end{aligned}
$$


By taking the $L^{1}$ norm in $\xi$ to (2.8) and using Young's inequality, we have

$$
\|v\|_{\mathscr{X}^{1}} \lesssim\left\|v_{0}\right\|_{X^{-1}}+\|v\|_{\mathscr{X}^{1}}\|v\|_{\mathscr{X}^{-1}} .
$$

Step 3. Combining (2.5) and (2.9), we finally have

$$
\|v\|_{\mathscr{X}^{-1}}+\|v\|_{\mathscr{X}^{1}} \lesssim\left\|v_{0}\right\|_{X^{-1}}+\left(\|v\|_{\mathscr{X}^{-1}}+\|v\|_{\mathscr{X}^{1}}\right)^{2},
$$

which implies the existence of a global-in-time solution in $\mathscr{X}^{-1} \cap \mathscr{X}^{1}$ for small data in $X^{-1}$.

\section{Proof of Theorem 1.3}

The proof of Theorem 1.3 is identical to the proof in [16], where Lemarié-Rieusset proved analyticity of the solution constructed by Le Jan-Sznitman [17:

$$
\sup _{0<t<\infty} \sup _{\xi \in \mathbb{R}^{3}} e^{\sqrt{t}|\xi|}|\xi|^{2}|\widehat{v}(t, \xi)|<\infty .
$$

Here we provide details of the proof of Theorem 1.3 for the reader's convenience. Again, we provide only a priori estimate (1.8) and we skip the iteration step. Let

$$
\widehat{V}(t, \xi):=e^{\sqrt{t}|\xi|} \widehat{v}(t, \xi) .
$$

Then, $\widehat{V}(t, \xi)$ satisfies that

$$
\begin{aligned}
\widehat{V}(t, \xi) & =e^{\sqrt{t}|\xi|-t|\xi|^{2}} \widehat{v_{0}}(\xi) \\
& -\int_{0}^{t}\left[e^{\sqrt{t}|\xi|-(t-s)|\xi|^{2}} \xi \cdot\left(\int_{\mathbb{R}^{3}} \widehat{v}(s, \xi-\eta) \widehat{v}(s, \eta) d \eta\right)\right] d s .
\end{aligned}
$$

Since $e^{\sqrt{t}|\xi|-\frac{1}{2} t|\xi|^{2}}$ is uniformly bounded in time, the linear term behaves like the linear term of (2.1). Thus, we only focus on the nonlinear term:

$$
\begin{aligned}
& \int_{0}^{t}\left[e^{\sqrt{t}|\xi|-(t-s)|\xi|^{2}} \xi \cdot\left(\int_{\mathbb{R}^{3}} \widehat{v}(s, \xi-\eta) \widehat{v}(s, \eta) d \eta\right)\right] d s \\
& =\int_{0}^{t}\left[e^{\left(\sqrt{t}|\xi|-\sqrt{s}|\xi-| \frac{1}{2}(t-s)|\xi|^{2}\right)} e^{-\frac{1}{2}(t-s)|\xi|^{2}} \xi \cdot e^{\sqrt{s}|\xi|}\left(\int_{\mathbb{R}^{3}} \widehat{v}(s, \xi-\eta) \widehat{v}(s, \eta) d \eta\right)\right] d s \\
& =\int_{0}^{t}\left[e^{\left(\sqrt{t}|\xi|-\sqrt{s}|\xi-| \frac{1}{2}(t-s)|\xi|^{2}\right)} e^{-\frac{1}{2}(t-s)|\xi|^{2}} \xi\right. \\
& \left.\cdot\left(\int_{\mathbb{R}^{3}} e^{\sqrt{s}(|\xi|-|\xi-\eta|-|\eta|)} \widehat{V}(s, \xi-\eta) \widehat{V}(s, \eta) d \eta\right)\right] d s
\end{aligned}
$$

Since $e^{\left(\sqrt{t}|\xi|-\sqrt{s}|\xi-| \frac{1}{2}(t-s)|\xi|^{2}\right)}$ and $e^{\sqrt{s}(|\xi|-|\xi-\eta|-|\eta|)}$ are uniformly bounded independently of $s$ and $t$, (3.1) can be bounded as

$$
\begin{aligned}
|\widehat{V}(t, \xi)| & \lesssim e^{-\frac{1}{2}|\xi|^{2}}\left|\widehat{v_{0}}(\xi)\right| \\
& +\int_{0}^{t}\left[e^{-\frac{1}{2}(t-s)|\xi|^{2}}|\xi|\left(\int_{\mathbb{R}^{3}}|\widehat{V}(s, \xi-\eta)||\widehat{V}(s, \eta)| d \eta\right)\right] d s .
\end{aligned}
$$

Therefore, we can obtain (1.8) by following the proof of Theorem 1.2 line by line. This completes the proof of Theorem 1.3 


\section{REFERENCES}

[1] Hantaek Bae, Animikh Biswas, and Eitan Tadmor, Analyticity and decay estimates of the Navier-Stokes equations in critical Besov spaces, Arch. Ration. Mech. Anal. 205 (2012), no. 3, 963-991, DOI 10.1007/s00205-012-0532-5. MR2960037

[2] Animikh Biswas and David Swanson, Gevrey regularity of solutions to the 3-D Navier-Stokes equations with weighted $l_{p}$ initial data, Indiana Univ. Math. J. 56 (2007), no. 3, 1157-1188, DOI 10.1512/iumj.2007.56.2891. MR2333469 (2008m:35261)

[3] Marco Cannone, Ondelettes, paraproduits et Navier-Stokes (French), Diderot Editeur, Paris, 1995. With a preface by Yves Meyer. MR 1688096 (2000e:35173)

[4] M. Cannone and F. Planchon, Self-similar solutions for Navier-Stokes equations in $\mathbf{R}^{3}$, Comm. Partial Differential Equations 21 (1996), no. 1-2, 179-193, DOI 10.1080/03605309608821179. MR1373769 (97a:35172)

[5] Jean-Yves Chemin, Théorèmes d'unicité pour le système de Navier-Stokes tridimensionnel (French), J. Anal. Math. 77 (1999), 27-50, DOI 10.1007/BF02791256. MR1753481 (2001c:35185)

[6] L. Escauriaza, G. Serigin, V. Sverak, $L_{3, \infty}$ solutions of Navier-Stokes equations and backward uniquness, Uspekhi Mat. Nauk, 58 (2003), 3-44.

[7] C. Foias and R. Temam, Gevrey class regularity for the solutions of the Navier-Stokes equations, J. Funct. Anal. 87 (1989), no. 2, 359-369, DOI 10.1016/0022-1236(89)90015-3. MR 1026858 (91a:35135)

[8] Hiroshi Fujita and Tosio Kato, On the Navier-Stokes initial value problem. I, Arch. Rational Mech. Anal. 16 (1964), 269-315. MR0166499 (29 \#3774)

[9] Giulia Furioli, Pierre G. Lemarié-Rieusset, and Elide Terraneo, Unicité dans $L^{3}\left(\mathbb{R}^{3}\right)$ et d'autres espaces fonctionnels limites pour Navier-Stokes (French, with English and French summaries), Rev. Mat. Iberoamericana 16 (2000), no. 3, 605-667, DOI 10.4171/RMI/286. MR.1813331 (2002j:76036)

[10] Pierre Germain, Nataša Pavlović, and Gigliola Staffilani, Regularity of solutions to the NavierStokes equations evolving from small data in $\mathrm{BMO}^{-1}$, Int. Math. Res. Not. IMRN 21 (2007), Art. ID rnm087, 35, DOI 10.1093/imrn/rnm087. MR2352218(2009d:76034)

[11] Zoran Grujić and Igor Kukavica, Space analyticity for the Navier-Stokes and related equations with initial data in $L^{p}$, J. Funct. Anal. 152 (1998), no. 2, 447-466, DOI 10.1006/jfan.1997.3167. MR.1607936 (99b:35168)

[12] Rafaela Guberović, Smoothness of Koch-Tataru solutions to the Navier-Stokes equations revisited, Discrete Contin. Dyn. Syst. 27 (2010), no. 1, 231-236, DOI 10.3934/dcds.2010.27.231. MR2600769 (2011d:35371)

[13] Tosio Kato, Strong $L^{p}$-solutions of the Navier-Stokes equation in $\mathbf{R}^{m}$, with applications to weak solutions, Math. Z. 187 (1984), no. 4, 471-480, DOI 10.1007/BF01174182. MR760047 (86b:35171)

[14] Herbert Koch and Daniel Tataru, Well-posedness for the Navier-Stokes equations, Adv. Math. 157 (2001), no. 1, 22-35, DOI 10.1006/aima.2000.1937. MR.1808843 (2001m:35257)

[15] Zhen Lei and Fanghua Lin, Global mild solutions of Navier-Stokes equations, Comm. Pure Appl. Math. 64 (2011), no. 9, 1297-1304, DOI 10.1002/cpa.20361. MR2839302 (2012k:35391)

[16] P. G. Lemarié-Rieusset, Recent developments in the Navier-Stokes problem, Chapman \& Hall/CRC Research Notes in Mathematics, vol. 431, Chapman \& Hall/CRC, Boca Raton, FL, 2002. MR.1938147 (2004e:35178)

[17] Yves Le Jan and Alain Sol Sznitman, Cascades aléatoires et équations de Navier-Stokes (French, with English and French summaries), C. R. Acad. Sci. Paris Sér. I Math. 324 (1997), no. 7, 823-826, DOI 10.1016/S0764-4442(97)86952-2. MR1446587 (97m:60123)

[18] Hideyuki Miura and Okihiro Sawada, On the regularizing rate estimates of Koch-Tataru's solution to the Navier-Stokes equations, Asymptot. Anal. 49 (2006), no. 1-2, 1-15. MR.2260554 (2007j:35160)

[19] Marcel Oliver and Edriss S. Titi, Remark on the rate of decay of higher order derivatives for solutions to the Navier-Stokes equations in $\mathbf{R}^{n}$, J. Funct. Anal. 172 (2000), no. 1, 1-18, DOI 10.1006/jfan.1999.3550. MR.1749867 (2000m:35147) 
[20] Fabrice Planchon, Asymptotic behavior of global solutions to the Navier-Stokes equations in $\mathbf{R}^{3}$, Rev. Mat. Iberoamericana 14 (1998), no. 1, 71-93, DOI 10.4171/RMI/235. MR 1639283 (99k:35144)

Department of Mathematics, University of California Davis, Davis, California 95616 E-mail address: hantaek@math.ucdavis.edu 